\section{Using Iodine Vapour Staining to Visualize Starch Distribution in a Dry, Extruded Food}

\author{
Jacquie Bond ${ }^{1}$ and Allan K Hardacre ${ }^{2}$ \\ ${ }^{1}$ Scion Research Ltd, Rotorua, ${ }^{2}$ New Zealand Institute for Crop \& \\ Food Research Ltd, Palmerston North, New Zealand \\ HardacreA@crop.cri.nz
}

\section{Introduction}

Food ingredients are often made by extruding flours and starches derived from cereal grains with proteins. Extrusion is carried out at high temperature, high shear and low water conditions to make low density, foamed pellets that are used as food ingredients. The structure of the crisp matrix varies greatly with the ingredient proportion and is important in forming the organoleptic properties of the crisps. However, it is very difficult to analyse the structure of the matrix with respect to the distribution of the major components, and the authors have been unable to find references for these materials. Furthermore, the materials must be stained without wetting as water will destroy the integrity of the crisps.

Spaghetti is a product in which starch granules have been extrusion cooked in a protein matrix before drying and so it is similar to dry extruded products, including whey crisps. A range of light and electron microscopy techniques have been described for spaghetti (Cunin et al., 1995; Heneen and Brismar, 2003). However, in many of the SEM images presented in that work, particularly where the starch granules had been damaged or gelatinised, it is difficult to resolve starch-rich regions in the spaghetti matrix.

In general, when SEM is used to analyse a sample, information comes from differences in the surface topography of either the cut or fractured surface, in which case, secondary electrons are imaged in LEI (lower electron imaging) mode. Differences in elemental composition, detected as atomic number, can be imaged in BSE (backscattered electron) mode. Crisps, such as those used in this work, are made up of aggregates of different materials but with similar molecular composition that present uniform or ambiguous topographical features in LEI and BSE modes. As a result, the images yield little in the way of useful information on how the ingredients are associated. Similar problems occurred when resolving the starch protein boundaries in SEM micrographs of spaghetti (Cunin et al., 1995; Dang and Copeland, 2004) and whey crisps (Matthey and Hanna, 1997; Onwulata et al., 2001; Singh et al., 1991) as the starch was gelatininsed.

Elemental iodine has an appreciable vapour pressure and is a weak general stain for biological material although it is best known as a specific stain with a strong affinity for starch. During the classic staining procedure using an iodine/potassium iodide solution, iodine binds inside the starch helix, resulting in an intense dark blue colour in the case of amylose or a reddish brown for amylopectin (Saenger, 1984). The iodine (in the form of $\mathrm{I}_{5}{ }^{-}$ions) is bound in the lumen of the coils of condensed sugar molecules that comprise the starch molecules.

In this paper we describe a method of SEM imaging that uses iodine vapour staining of starch to reveal the distribution of starch and protein in dry extruded crisps made from starch and dairy protein blends. The method was simple and provided excellent resolution of starch-rich and starch-poor regions.

\section{Methods and materials}

Extrusion. The crisps used for this work were produced by extruding a mixture of dairy protein and rice flour using a Clextral BC21 twin screw extruder (Clextral, Riez 42702 Firminy Cedex France) located at Massey University, Palmerston North. The extruder was fitted with a die with two $2 \mathrm{~mm}$ diameter holes in conjunction with a variable speed,

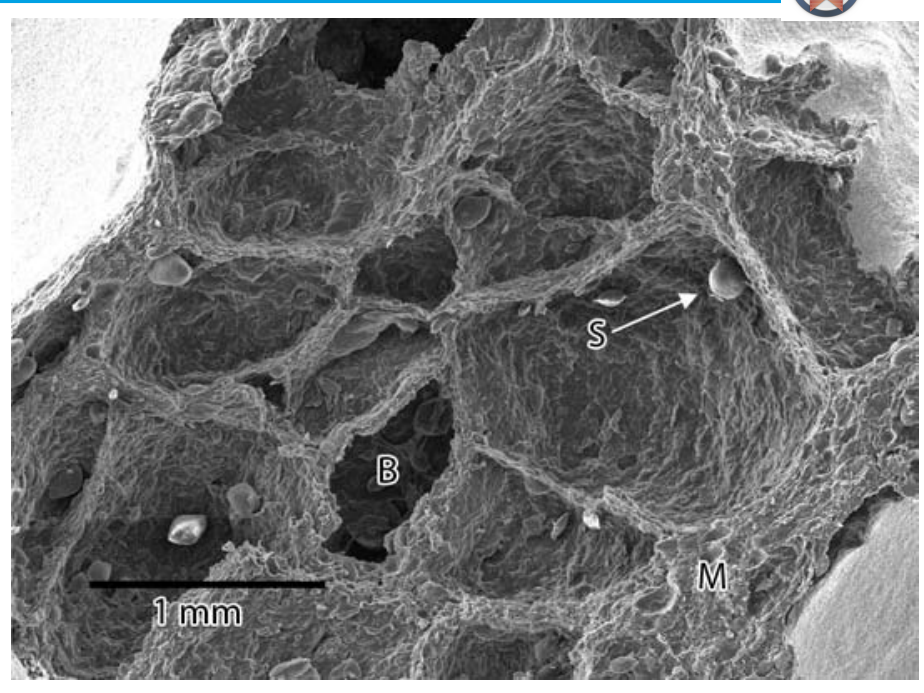

Fig 1. Sectioned whey crisp, $55 \times$, LEI mode, showing the strongly textured matrix material. $B=$ Bubble, $M=$ matrix material, $S=$ Starch granule.

rotating knife that cut the emerging extrusion into pellets (crisps). The extruder was operated at $90^{\circ} \mathrm{C}$ during processing.

The crisps were made from a mixture of rice flour (Remy R200T, Invita NZ Ltd Box 58-843, Greenmount, Auckland) and whey protein isolate (Fonterra, WPI 894, Fonterra NZ, Private Bag 92032 Auckland 1142 ) blended to give a final protein content of $50 \%$ calculated from proximate analyses of the ingredients. Water was added to facilitate the manufacturing process and to give a final moisture content of about $23 \%$ as the crisps left the extruder. The crisps were dried at $35^{\circ} \mathrm{C}$ in a forced draft oven for 24 hours to $10 \%$ moisture content (wet weight basis, $\mathrm{wwb}$ ) and stored at room temperature in sealed bags before staining and examination with the SEM. The final product was a crisp dry pellet approximately $3 \mathrm{~mm}$ in diameter with a foam-like internal structure (Fig 1). The internal surface of the bubbles within the foam is strongly textured, suggesting that the solid matrix is not of uniform composition. Starch granules that survived the extrusion process can be seen on the walls of bubbles. Many of the features seen in Fig 1 are described for spaghetti (Cunin et al., 1995)

Depending on the conditions of extrusion, the fractured surface of the solid matrix may appear rough (Fig 1), giving the impression of particles embedded in a matrix or glassy and uniform (Fig 2a). In both products the highly textured bubble walls are a common feature.

Staining Iodine vapour diffusion was carried out in closed glass jars. About 10 crisps that had been sectioned or fractured with a sharp blade were placed in a small dish within the jar along with another dish that contained about $5 \mathrm{~g}$ of iodine flakes.

Electron microscopy A JEOL JSM 6700 scanning electron microscope (JEOL- Ltd. 1-2, Musashino 3-chome, Akishima Tokyo 196-8558, Japan) was used in LEI (lower electron imaging) mode for surface topography and BSE (back scattered electron) mode to enhance differences in atomic number. The crisps were attached to stubs, fracture face uppermost, with silver paint and were thinly coated with chromium before the fractured faces were imaged. The LEI and BSE images were made at $15 \mathrm{kv}$ with a probe current of $10 \mu \mathrm{A}$ and a working distance of $14.8 \mathrm{~mm}$.

\section{Results}

The effect of time of exposure of the crisps to iodine vapour is shown in Fig 2 (a-d)

Iodine vapour staining does not alter the structure of the crisp as would occur with water-based stains; therefore the sample is maintained in its native form. Exposing the crisps to iodine vapour reveals clear regions of high and low affinity for iodine; regions that bind iodine are 
EMS offers the breakthrough solutions of Quantomik's proprietary WETSEM ${ }^{\circledR}$ Technology now availailite at hali price or a limiced time
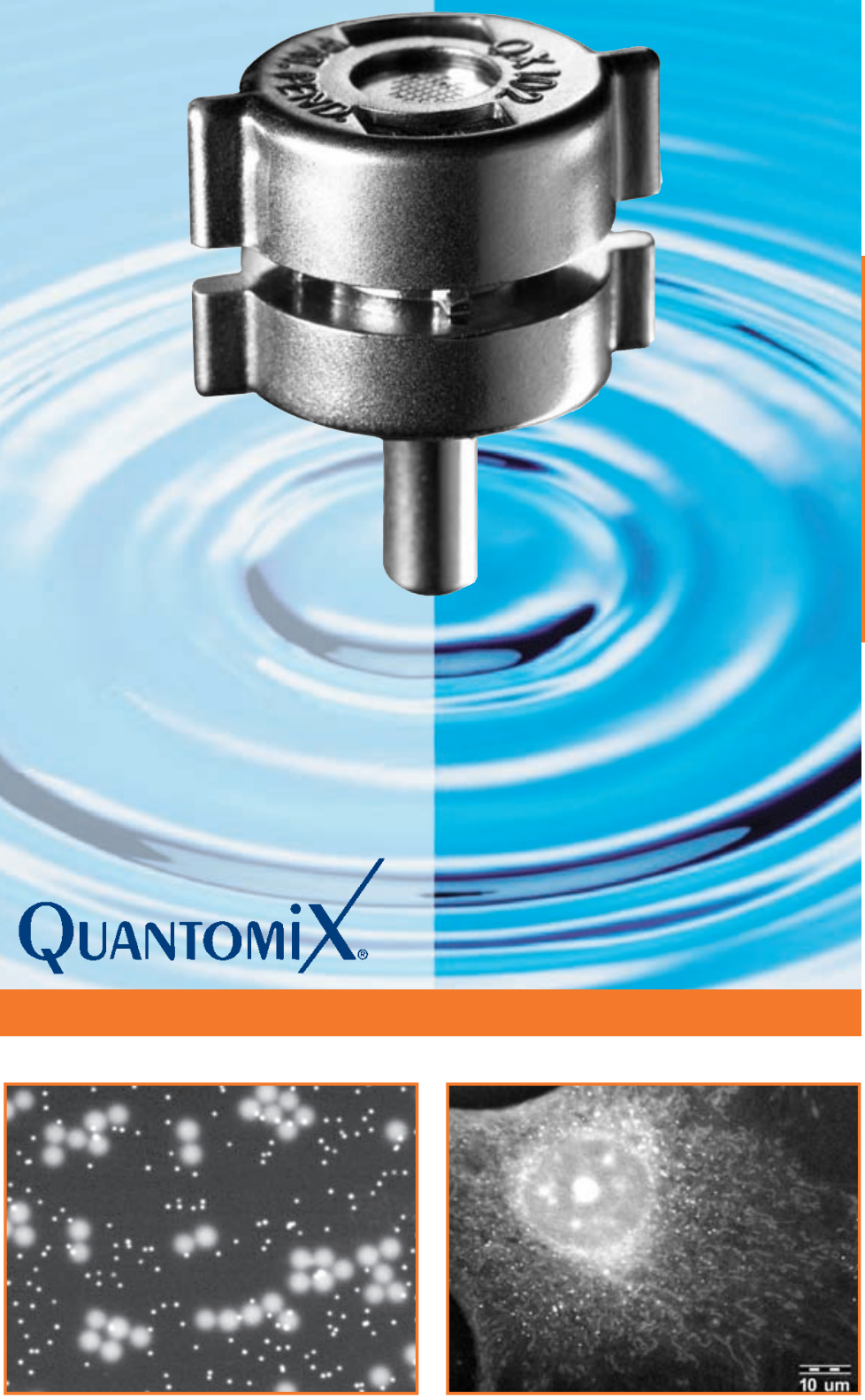

Silica $(500 \mathrm{~nm})$ and gold $(40 \mathrm{~nm})$ particles

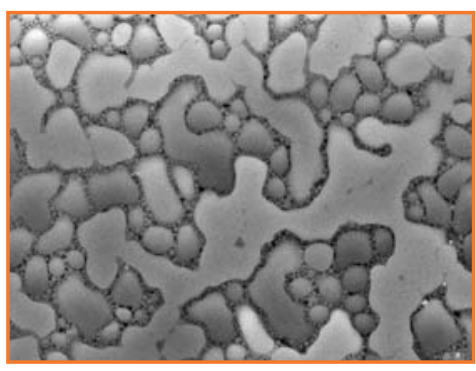

Volatile deodorant showing phase separation

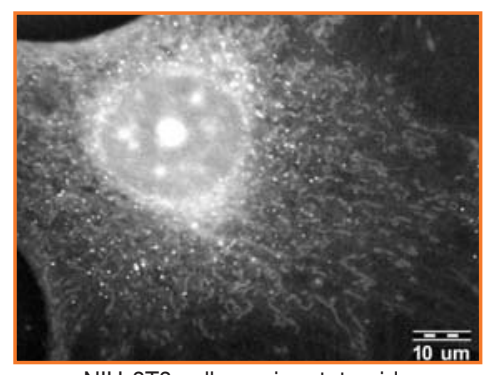

$\mathrm{NIH}-3 \mathrm{~T} 3$ cell, osmium tetroxide

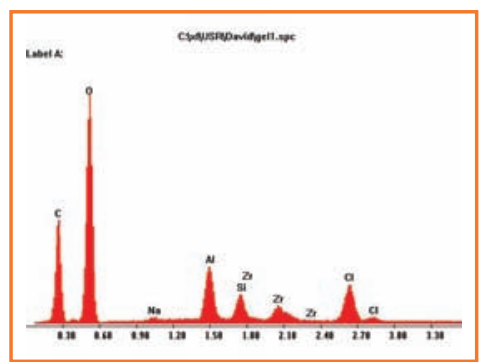

EDS graph showing deodorant material composition
The biggest breakthrough is here in a nano-package.

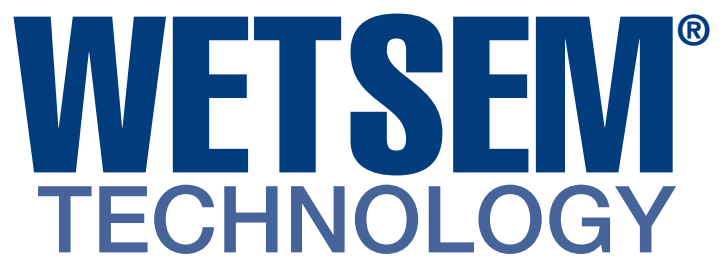

Attain EM-level imaging of wet samples

Reduce artifacts \& time-consuming sample preparation

Safe and simple to use

Achieve reliable, reproducible \& quantifiable results

\section{Applications}

- Industrial research: food, oils, dyes, emulsions, pharmaceuticals, suspensions, personal care goods, cosmetics, inks.

- Quality control and quality assurance.

- Life sciences and medicine: cultured and primary cells, histology, nerve cells and myelin imaging, microbiology, plants.

- Tissue implants and prostheses.

- Environmental and toxicological applications.

- Clinical diagnosis: histopathology, cytology, oncology.

\section{Capabilities}

- Analyze size distribution, aggregation and homogeneity of particle suspensions.

- Perform EDS of fully hydrated samples.

- SEM imaging of oils, greases, volatile substances, emulsions and creams.

- Enables tissue morphology and analysis.

- Characterize cellular and subcellular organelles, cell contacts and receptors, cytoskeleton and other detail.

- Characterize and quantify lipids in fully-wet cells and tissues.

Please contact us for more detaills.

Electron Microscopy Sciences In PA: (215) 412-8400 • Toll-Free (800) 523-5874

Fax (215) $412-8450$ or 8452

email: sgkcck@aol.com or stacie@ems-secure.com

www.emsdiasum.com 


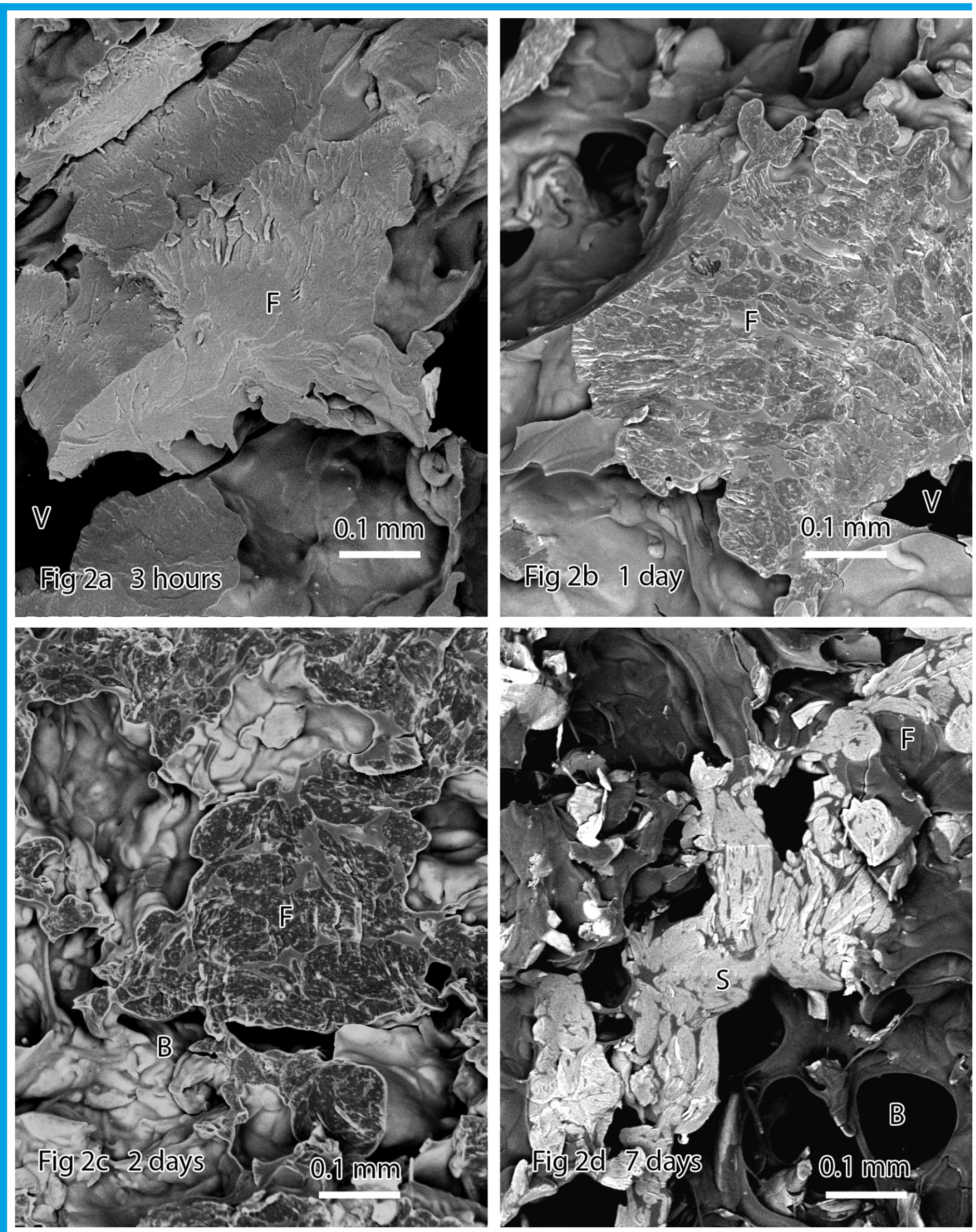

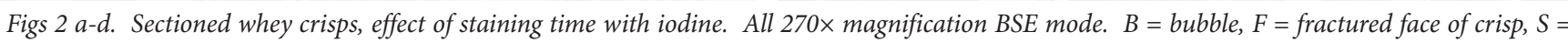
starch rich region. 


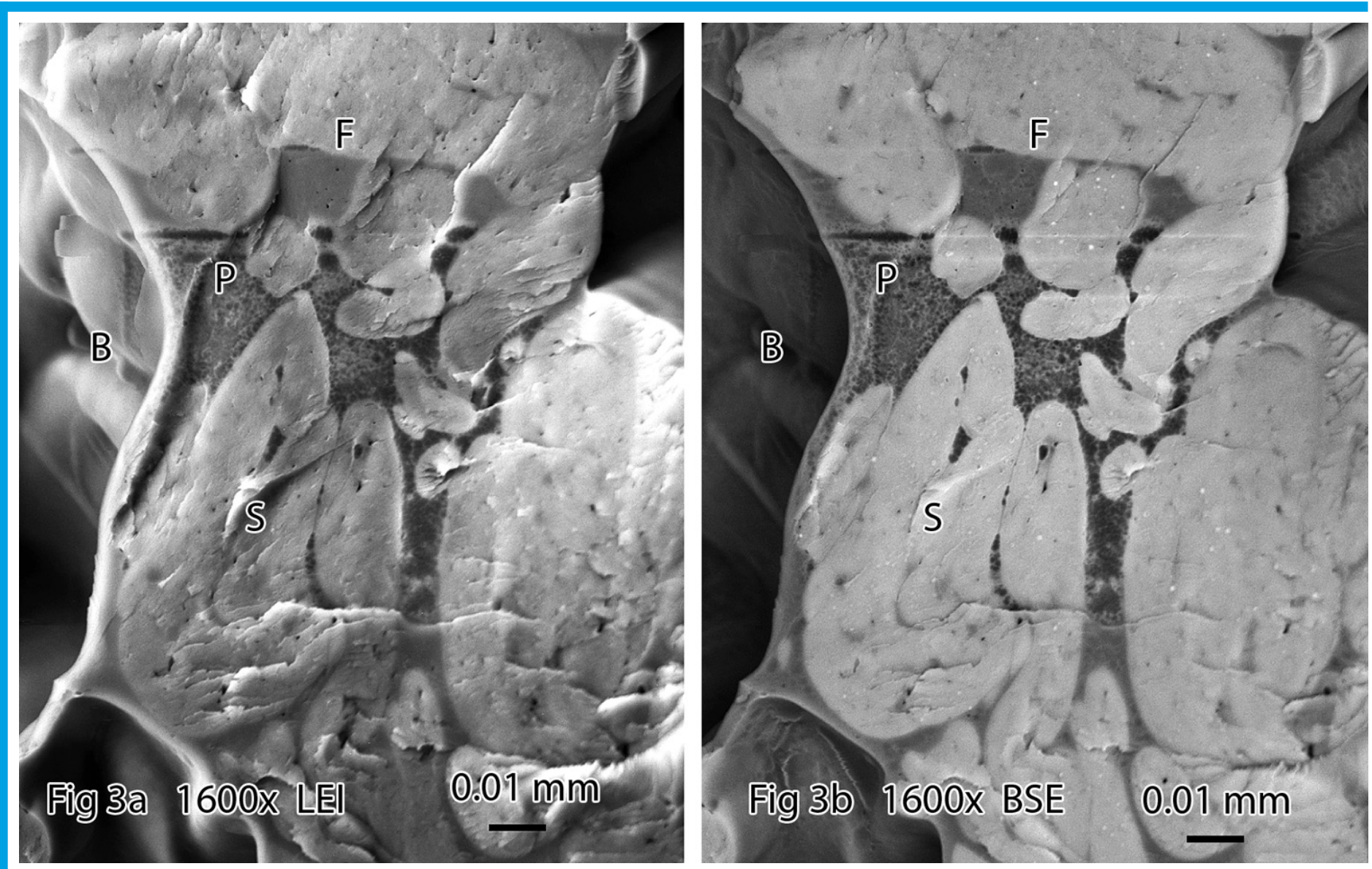

Fig 3 a,b Sectioned whey crisps stained with iodine. $1600 \times$ magnification BSE mode. $B=$ bubble, $F=$ fractured face of crisp, $P=$ protein-rich region, $S=$ starch-rich region.

bright while the darker regions contain less iodine.

After 3 hours' exposure to iodine, the sample appears uniform with no change in brightness across the fractured surface. After 1 day (Fig 2b) small differences in brightness are evident over the surface and these intensified by day 2 (Fig 2c). The highly granular protrusions on the bubble walls appear to have a lighter "skin" and a darker centre. In the final image made after 7 days' exposure to iodine the contrast in the image is reversed and large agglomerations of iodine-rich material are evident within the matrix material.

We suggest that the iodine first binds at low concentration to the protein component of the crisps but, with time, diffusion into the starch component continues, eventually resulting in the bright, iodine-rich starch areas in the image in Fig 2d.

At higher magnification $(1600 \times)$, crisps exposed to iodine vapour for 7 days clearly show large variations in image brightness that are associated with structures in the crisp matrix. Imaged in LEI mode (Fig $3 a)$, the relatively uniform nature of the fractured surface is evident and, clearly, fracture planes are not associated with any of these structures indicating a relatively uniform glassy material.

When the same region is imaged in BSE mode (Fig 3b) more detail appears. The bright iodine-stained, starch-rich areas appear as inclusions within a protein-rich film that coats the inside of the bubbles and permeates spaces within the starch-rich regions. The protein-rich areas have a granular structure. The brighter regions or stippling within the protein-rich areas may be amylose leached from the starch granules during processing. Clearly, the starch and protein components do not form a uniformly dispersed material even during high shear processing in an extruder.

\section{Conclusions}

This work shows that iodine vapour staining is a useful simple and easy to use technique for selectively staining starchy inclusions in pro- teinacious matrices. It may also have useful applications in visualising the distribution of starch in products where starch is intimately mixed with ingredients of different affinity for iodine. The technique provided excellent contrast between ingredients that were impossible to distinguish using other techniques.

It was evident from this work that the starch component did not form a homogenous dispersion with the protein during the extrusion of these materials.

\section{Acknowledgements}

This work was carried out as part of the New Zealand governmentfunded contract DRIX0201. The financial support of the Foundation for Research, Science and Technology is gratefully acknowledged.

\section{References}

Cunin, C., S. Handschin, P. Walther, and E. Escher. 1995. Structural changes of starch during cooking of durum wheat pasta. Lebensmittel-Wissenschaft und -Technologie Food Science and Technology 28:323-328.

Dang, J.M.C., and L. Copeland. 2004. Studies of the fracture surface of rice grains using environmental scanning electron microscopy. J Sci Food Agric 84:707-713.

Heneen, W.K., and K. Brismar. 2003. Structure of cooked spaghetti of durum and bread wheats. Starch/Staerke 55:546-557.

Matthey, F.P., and M.A. Hanna. 1997. Physical and functional properties of twinscrew extruded whey protein concentrate corn starch blends. Lebensm-Wiss Technol 30:359-366.

Onwulata, C.I., P.W. Smith, R.P. Konstance, and V.H. Holsinger. 2001. Incorporation of whey products in extruded corn, potato or rice snacks. Food Research International 34:679-687.

Saenger, W. 1984. The structure of the blue starch-iodine complex. Naturwissenschaften 71:31-36.

Singh, R.K., S.S. Nielson, and J.V. Chambers. 1991. Selected characteristics of extruded blends of milk protein raffinate or nonfat dry milk with cornflour. Journal of Food Processing and Preservation 15:285-302. 